\title{
ON BANACH LATTICE ALGEBRAS
}

\author{
Ruşen YILMAZ ${ }^{1}$ AND YILMAZ ALTUN ${ }^{2}$ \\ ${ }^{1}$ Department of Mathematics, Recep Tayyip Erdog̃an University, 53100 Rize, TURKEY \\ rusen.yilmaz@erdogan.edu.tr, ryilmaz00@yahoo.com \\ 2 Department of Mathematics, Artvin Coruh University, 08100 Artvin, TURKEY \\ yilmazaltun@artvin.edu.tr
}

\begin{abstract}
In this paper we investigate a characterization of a Banach lattice algebra with unit to be represented as an AM-f-algebra. We also consider, for a locally compact Hausdorff topological space $L$ and a Banach lattice space $A$, the identification of $C_{b}(L, Z(A))$ with the center $Z\left(C_{0}(L, A)\right)$ of $C_{0}(L, A)$.
\end{abstract}

Keywords: Lattice Ordered Algebra; $\ell$-algebra; Riesz Algebra; $f$-algebra; Banach Lattice Algebra; Banach $f$-algebra; $A M$-space.

1. Introduction. The main result of this paper is the representation of a Banach lattice algebra with unit as an algebra of continuous functions on a compact Hausdorff topological space $K$, with the usual partial ordering, pointwise multiplication $\cdot$, and the supremum norm; that is, $(A, *) \cong(C(K), \cdot)$, where $*: A \times A \rightarrow A$ is the algebraic multiplicative operator in a Banach lattice algebra $A$. The results in this direction were given by Martignon in [15]. In this paper we improve her results and prove that, under certain conditions, $(A, *)$ and $(C(K), \cdot)$ are isometrically and algebraically $\ell$-isomorphic as $A M$ - $f$-algebras. We also attempt to clarify some results in the same direction due to Ercan and Wickstead [7]. In particular, we prove that, if $A$ is a Banach lattice space and $L$ a locally compact Hausdorff space, the center of the $\ell$-space $C_{0}(L, A)$ of all continuous $A$-valued functions on $L$ is isometrically isomorphic to the space $C_{b}(L, Z(A))$ of all norm bounded $Z(A)$-valued functions on $L$, where $Z(A)$ is the center of $A$ endowed with the strong operator topology.

\section{Preleminaries.}

Definition 2.1. A real lattice ordered linear space ( $\ell$-space) $A$ is said to be a lattice ordered algebra (an $\ell$-algebra or a Riesz algebra) if it is a linear algebra (not necessarily associative) such that if $a, b \in A^{+}$, then $a b \in A^{+}$. An $\ell$-algebra $A$ is said to be

(i) an $f$-algebra (function algebra) if $a \wedge b=0$ implies $a c \wedge b=c a \wedge b=0$ for all $c \in A^{+}$, (although the following classes of $\ell$-algebras are not explicitly used, we include their definitions for the sake of completeness)

(ii) an almost $f$-algebra if $a \wedge b=0$ implies $a b=0$, 
(iii) a d-algebra if $c(a \vee b)=c a \vee c b$ and $(a \vee b) c=a c \vee b c$ for all $a, b \in A$ and $c \in A^{+}$.

The notion of an $f$-algebra, as given in the above definition, first appeared in a paper by Birkhoff and Pierce [5] in 1956 to be followed a decade later by the class of almost $f$-algebras introduced by Birkhoff in [4]. The notion of a $d$-algebra was introduced by Kudláček [13] in 1962. In general, these classes of algebras are distinct, but there are relations between them; for example, it is clear that every $f$-algebra is an almost $f$-algebra and a $d$-algebra. Every Archimedean (that is, for all $a, b \in A^{+}$and $n=1,2, \ldots, n a \leq b$ implies $\left.a=0\right) f$-algebra is commutative and associative. It turns out that every Archimedean almost $f$-algebra is commutative but not necessarily associative. In any semi-prime associative $\ell$-algebra the classes of $f$-algebras, almost $f$-algebras and $d$-algebras are equivalent. In particular, this holds for an associative $\ell$-algebra with (algebraic) unit element $e>0$. We refer the reader to [3] for details of these results as given by Bernau and Huijmans.

For the elementary theory of $\ell$-space and terminology not explained here we refer to $[2,14,16,20]$.

Let $E$ and $F$ be $\ell$-spaces. A linear operator $T: E \rightarrow F$ is said to be order bounded if the image under $T$ of an order bounded set in $E$ is again an order bounded set in $F$. The operator $T$ is called positive $T\left(E^{+}\right) \subset F^{+}$. A linear operator $T: E \rightarrow F$ is called an $\ell$-homomorphism (or a Riesz homomorphism) whenever $a \wedge b=0$ in $E$ implies $T a \wedge T b=0$. Clearly, every $\ell$-homomorphism is positive. An order bounded linear operator $\pi: E \rightarrow E$ is called an orthomorphism if and only if, for all $a, b \in E, a \perp b$ implies $\pi a \perp b$. The collection of all orthomorphisms on $E$ is denoted by $\operatorname{Orth}(E)$. Obviously every positive orthomorphism is an $\ell$-homomorphism. It is well-known that the ordered vector space $\mathcal{L}_{b}(E, F)$ of all order bounded linear mappings of an $\ell$-space $E$ into a Dedekind complete $\ell$-space $F$ is a Dedekind complete $\ell$-space (see, for example, [2, Theorem 1.13]). In the case that $E=F, \mathcal{L}_{b}(E, F)$ is denoted by $\mathcal{L}_{b}(E)$. It is also well-known that, if $E$ is an (Archimedean) $\ell$-space, then $\operatorname{Orth}(E)$ is an (Archimedean) $f$-algebra under multiplication by composition, possessing the identity operator $I$ on $E$ as a multiplicative identity (see [2]), and we recall that $\operatorname{Orth}(E)$ is an $f$-subalgebra of $\mathcal{L}_{b}(E)$.

The notion of an orthomorphism is related to that of a multiplier. An order bounded operator $T: E \rightarrow E$ is said to be a multiplier on an $\ell$-algebra $E$ if $T(a b)=(T a) b=a(T b)$ for all $a, b \in E$. The algebra of all multipliers on $E$ is denoted by $M(A)$. If $E$ is an Archimedean $f$-algebra, then every orthomorphism $\pi: E \rightarrow E$ is a multiplier; that is $\operatorname{Orth}(E) \subseteq M(E)$. Indeed, for each $a \in E$, the operator $\pi_{a}$ defined by $\pi_{a}(b)=a b(b \in E)$ is an orthomorphism, and so,

$$
\pi(a b)=\pi\left(\pi_{a} b\right)=\left(\pi \pi_{a}\right) b=\left(\pi_{a} \pi\right) b=a \pi b .
$$

If $E$ is an Archimedean semi-prime $f$-algebra, then $\operatorname{Orth}(E)=M(E)$ [18].

Definition 2.2. Let $A$ be an $\ell$-space. The solid subspace generated by the identity operator $I$ in $\mathcal{L}_{b}(A)$ is said to be the center of $A$ and is denoted by $Z(A)$; that is,

$$
Z(A)=\left\{T \in \mathcal{L}_{b}(A):|T| \leq n I \text { for some } n \in I N\right\} .
$$

The center $Z(A)$ of an $\ell$-space $A$ is an $\ell$-subalgebra of $\operatorname{Orth}(A)$. To see this, we first note that $Z(A)$ is itself an $\ell$-space since $Z(A)$ is a solid subspace. Moreover, 
it can easily be seen that $0 \leq S T \in Z(A)$ for all $0 \leq S, T \in Z(A)$; that is, $Z(A)$ is itself an $\ell$-algebra. So it is sufficient to show that $a \perp b$ in $A$ implies that $T a \perp b$ in $A$ for all $T \in Z(A)$. Observe that $|T a| \leq|T||a|$ for all $T \in \mathcal{L}_{b}(A)$ and $a \in A$. Hence, for $T \in Z(A)$,

$$
\begin{aligned}
|T a| \wedge|b| & \leq|T||a| \leq n I|a| \wedge|b| \quad(\text { some } n \in I N) \\
& =n|a| \wedge|b| \leq n(|a| \wedge|b|) \quad(\text { all } n \in I N) \\
& =0
\end{aligned}
$$

that is, $T a \perp b$. It is also obvious that $T$ is order bounded, from which follows that $T \in \operatorname{Orth}(A)$. Moreover, we see that, for all $T \in \operatorname{Orth}(A)$ and $a \in A^{+},|T a|=$ $|T| a \leq n I a=n a$ for some $n \in I N$ (note that $|T a|=|T||a|$ for all $T \in \operatorname{Orth}(A)$ and $a \in \bar{A}$; see, for instance, [2, Thoerem 8.6]). Therefore the above definition may be refined as follows,

$$
Z(A)=\left\{T \in \operatorname{Orth}(A):|T a| \leq n a \text { for some } n \in I N \text { and all } a \in A^{+}\right\} .
$$

Now it is obvious that $Z(A)$ of an Archimedean $\ell$-space $A$ is an Archimedean $f$ subalgebra of $\operatorname{Orth}(A)$, and so is necessarily commutative and associative.

A norm $\|\cdot\|$ on an $\ell$-space $A$ is said to be a lattice norm ( $\ell$-norm) if $|a| \leq|b|$ in $A$ implies $\|a\| \leq\|b\|$, and the pair $(A,\|\cdot\|)$ is called a normed lattice space (normed $\ell$-space). If a normed $\ell$-space $(A,\|\cdot\|)$ is norm complete, then $(A,\|\cdot\|)$ is referred to as a Banach lattice space (Banach $\ell$-space). A Banach $\ell$-space $(A,\|\cdot\|)$ is said to be an $A M$-space (abstract $M$-space) if $\|a \vee b\|=\|a\| \vee\|b\|$ holds for all $a, b \in A^{+}$.

We recall that every normed $\ell$-space is Archimedean and an orthomorphism $\pi$ on a normed $\ell$-space $A$ is norm bounded (i.e. $\|\pi a\| \leq\|\pi\|\|a\|$ for all $a \in A$ ) if and only if there exists a positive real number $\lambda$ such that $|\pi| \leq \lambda I$; that is, $\pi \in \operatorname{Orth}(A)$ is norm bounded if and only if $\pi \in Z(A)$ (for details see [19, §144]). Note that this statement indicates that $Z(A)$ consists precisely of all norm bounded orthomorphisms on $A$. Moreover, the operator norm $\|\cdot\|$ on $Z(A)$ is an $\ell$-norm and coincides with the Minkowski functional of $[-I, I]$

$$
\|T\|_{\infty}=\inf \{\lambda>0:|T| \leq \lambda I\}
$$

for all $T \in Z(A)$; more explicitly, $\|T\|=\|T\|_{\infty}$ for all $T \in Z(A)$.

In the special case when $A$ is a Banach $\ell$-space every orthomorphism on $A$ is norm bounded; that is, $\operatorname{Orth}(A)=Z(A)([17$, Corollary 4.2]). The Banach $\ell$-space $(\operatorname{Orth}(A),\|\cdot\|)$ is indeed a Banach $\ell$-algebra since the operator norm $\|\cdot\|$ on $\mathcal{L}_{b}(A)$ is an algebra norm, and it is an $\ell$-norm as $\|T\|=\|T\|_{\infty}$. Wickstead proved in [17] that, if $A$ is a Banach $\ell$-space, then $\operatorname{Orth}(A)$ is an $A M$-space with the identity operator $I$; in fact, $\operatorname{Orth}(A)$ is a Banach $f$-algebra since $\operatorname{Orth}(A)$ is an $f$-algebra itself.

Next we define a concept of an $A M$ - $f$-algebra.

Definition 2.3. A Banach $f$-algebra $(A,\|\cdot\|)$ with the property $\|a \vee b\|=\|a\| \vee\|b\|$ for all $a, b \in A^{+}$is called an $A M$-f-algebra; in other words, an $A M$-space which is also an $f$-algebra is called an $A M$ - $f$-algebra.

The space $C_{b}(X)$ of continuous bounded functions on a topological space $X$, and its closed $\ell$-subspaces, with the usual partial ordering, multiplication (pointwise) and the operator norm, are examples of $A M$ - $f$-algebras. 
We summarize our results in the following.

Theorem 2.4. If $A$ is a Banach $\ell$-space, then

$$
Z(A)=\operatorname{Orth}(A)=\left\{\pi \in \mathcal{L}_{b}(A):|\pi| \leq \lambda I \text { for some } \lambda>0\right\},
$$

and $\|\pi\|=\inf \{\lambda>0:|\pi| \leq \lambda I\}$ holds for all $\pi \in Z(A)$. In particular, $Z(A)$ under this norm is an $A M$-f-algebra with the identity operator $I$ as its multiplicative identity.

A positive element $u$ in a normed $\ell$-space $A$ is called a norm order unit if and only if $\|u\|=1$ and, for any $a \in A^{+}$, with $\|a\| \leq 1, a \leq u$. A positive element $u$ in an $\ell$-space $A$ is said to be an order unit if and only if, for every $a \in A$, there exists a positive integer $n$ depending upon $a$ such that $|a| \leq n u$ (or, equivalently, if and only if the solid subspace $S_{u}$ generated by $u$ is equal to $A$ ). Clearly, in a normed $\ell$-space every norm order unit is an order unit, and in an $\ell$-space every order unit is a weak order unit (A positive element $u$ in an $\ell$-space $A$ is said to be an weak order unit if $a \wedge u=0$ implies $a=0)$. Indeed, let $a \wedge u=0$. Then $0 \leq a \wedge n u \leq n(a \wedge u)=0$ for all $n \in I N$; that is, $a \wedge n u=0$ for all $n \in I N$. If $u$ is an order unit, then $0 \leq a \leq n u$ for some $n \in I N$, and so $a=a \wedge n u=0$, as required.

Every weak order unit need not be an order unit. For example, the $\ell$-space $C([0, \infty))$ has the weak order unit $u(x)=1(0 \leq x \leq \infty)$. However, $C([0, \infty))$ has no order unit; for, if $0 \leq f(x)=(u(x)+n x)^{2}$ in $C([0, \infty))$, then there exists no $n \in I N$ and $u(x) \geq 0$ such that $f(x) \leq n u(x)$ for all $0 \leq x<\infty$.

In general, if $A$ is a normed $\ell$-space with a norm order unit $u$, then $a \in A$ and $\|a\| \leq 1$ imply that $|a| \leq u$; for,

$$
\left\|a^{+}+a^{-}\right\|=\||a|\|=\|a\| \leq 1
$$

and so $a^{+}+a^{-} \leq u$ since $a^{+}+a^{-} \in A^{+}$; that is, $|a| \leq u$.

Remark 2.5. We note that the identity operator $I$ on a normed $\ell$-space $A$ is a norm order unit in $Z(A)$ (and hence in $\operatorname{Orth}(A)$ ), which is also a multiplicative identity. In fact, in general if $A$ is a normed $\ell$-algebra with multiplicative modulus and a multiplicative identity $e \geq 0$, then $e$ is the norm order unit of $A$ by 2.1. Lemma of $[15]$.

3. A Representation Theorem for an $A M$ - $f$-algebra. In this section we discuss necessary and sufficient conditions for a Banach $\ell$-algebra $(A, *)$ with unit to be represented as an algebra $(C(K), \cdot)$ of continuous real-valued functions on a compact Hausdorff topological space $K$, with the usual partial ordering, pointwise multiplication ', and the supremum norm. Investigations in this direction have been carried out by Martignon [15]. Here we improve her results and prove that, under certain conditions, $(A, *)$ and $(C(K), \cdot)$ are isometrically and algebraically $\ell$-isomorphic as $A M$-f-algebras.

It is well known that if $A$ is an Archimedean $\ell$-algebra with a multiplicative unit $e>0$ which is an order unit (even a weak unit), then it is an $f$-algebra ([3, Corallary 1.10.]). Moreover, if $A$ is equipped with two $f$-algebra multiplications with the same multiplicative unit, then these multiplications coincide on $A$. More precisely, if $A$ is an Archimedean $\ell$-space and $e>0$ in $A$, then there exists at most one product on $A$ 
that makes $A$ an $f$-algebra having $e$ as its multiplicative unit ([2, Theorem 8.23.]). Now combining these facts we obtain the following result, due to Martignon [15, 1.4. Proposition].

Theorem 3.1. Let $K$ be a compact Hausdorff topological space and suppose that $(C(K), *)$ is an $\ell$-algebra such that $1(x)=1$ for all $x \in K$. Furthermore, if 1 is the multiplicative identity of $(C(K), *)$, then $(C(K), *)=(C(K), \cdot)$; in other words, * is the pointwise multiplication $\cdot$

Suppose that $A$ is a Banach $\ell$-space with an order unit $u$. Then, by the definition of an order unit, $S_{u}=A$ holds, and so it follows from Theorem 12.20 of [2] that $A$, endowed with the norm

$$
\|a\|_{\infty}=\inf \{\lambda>0:|a| \leq \lambda u\}
$$

is an $A M$-space having the order interval $[-u, u]$ as its closed unit ball. Moreover, $\|a\|_{\infty}$ is equivalent to the original norm on $A$ since all $\ell$-norms making an $\ell$-space a Banach $\ell$-space are equivalent (see [2, Corollary 12.4]. Summarizing these results, we have

Theorem 3.2. If a Banach $\ell$-space $A$ has an order unit $u$, then $A$ can be remormed such that $A$ becomes an $A M$-space having $[-u, u]$ as its closed unit ball. In particular, the order unit $u$ is a norm order unit.

As observed before, every norm order unit in a normed $\ell$-space is an order unit. Thus, the preceding theorem yields the following characterization.

Corollary 3.3. If $u$ is a positive element in a Banach $\ell$-space, then $u$ is an order unit if and only if it is a norm order unit.

In the sequel, unless otherwise stated, we shall mean by the phrase " $A M$-space with unit" a Banach $\ell$-space with an order unit, whose norm is the $\|\cdot\|_{\infty}$-norm.

The following is an extension of Kakutani's Representation Theorem to AMalgebras with unit. We first note that Kakutani's Representation Theorem deals with $A M$-spaces with unit. This result is due to Kakutani [9, Theorems 2 and 21], and was later extended by M. Krein and S. Krein [10, 11]. We also note that an injective $\ell$-homomorphism $T$ from an $\ell$-space $A$ into an $\ell$-space $B$ is referred to as an $\ell$-isomorphism, and that $A$ and $B$ are said to be $\ell$-isomorphic if $T$ is also surjective.

Theorem 3.4. A Banach $\ell$-algebra $(A, *)$ with unit $u$ is an AM-algebra if and only if $(A, *)$ is isometrically and algebraically $\ell$-isomorphic to $(C(K), \cdot)$ for some (unique up to homeomorphism) compact Hausdorff topological space $K . K$ can be chosen to be the set of all algebraic $\ell$-functionals $f$ from $A$ into IR such that $f(u)=1$, endowed with the weak topology $\sigma\left(A^{\prime}, A\right)$.

In general, a Banach $\ell$-algebra $(A, *)$ is an AM-algebra if and only if $(A, *)$ is isometrically and algebraically $\ell$-isomorphic to a closed $\ell$-subalgebra of a $(C(K), \cdot)$ algebra.

Proof. A complete proof of the theorem can be found in [2, Theorem 12.28]. We give here a sketch of the proof. Suppose that $A=C(K)$ for a compact Hausdorff topological space $K$. Clearly, $A$ is an $A M$-algebra with unit the constant function 
1. We now discuss the description of $K$ in connection with the algebraic lattice structure of $A$. Set

$$
L=\left\{f \in\left(U^{\prime}\right)^{+}: f \text { is an extreme point of }\left(U^{\prime}\right)^{+} \text {with } f(u)=\|f\|=1\right\}
$$

and

$$
K=\left\{g \in\left(U^{\prime}\right)^{+}: g \text { is an algebraic } \ell \text {-functional with } g(u)=\|g\|=1\right\},
$$

where $U^{\prime}=\left\{h \in A^{\prime}:\|h\| \leq 1\right\}$, the closed unit ball of $A^{\prime}$. Now since $U^{\prime}$ is $\sigma\left(A^{\prime}, A\right)$ compact by Alaoglu's Theorem (see, for example, [6, $\S 3$ of Chapter V]), and so $L$ being $\sigma\left(A^{\prime}, A\right)$-closed in $U^{\prime}$ is also $\sigma\left(A^{\prime}, A\right)$-compact. Since $A$ is an $A M$-space, it follows from Theorem 12.27 of [2] that $K \subseteq L$. Therefore $K$ is $\sigma\left(A^{\prime}, A\right)$-compact and Hausdorff.

On the other hand, suppose that $A$ is an $A M$-algebra with unit $u$ and define the mapping $T: A \rightarrow C(K)$ by $T a(g)=g(a)$ for all $a \in A$ and $g \in K$. It follows from the Krein-Milman theorem (see [12] or [2, Theorem 9.14]) that $T$ is a norm preserving $\ell$-isomorphism. The fact that every element $g \in K$ is an algebraic homomorphism implies that $T$ is also an algebraic homomorphism. Indeed,

$$
T(a * b)(g)=g(a * b)=g(a) g(b)=(T a(g))(T b(g))=(T a \cdot T b)(g)
$$

holds for all $g \in K$, and so $T(a * b)=T a \cdot T b$ for all $a, b \in A$. Moreover, the algebraic $\ell$-isomorphism $T$ maps the unit element $u$ of $A$ onto the multiplicative identity 1 of $C(K)$ (the constant function 1 on $K$ ); for, $(T u)(g)=g(u)=1$ for all $g \in K$, and so $T u=1$. Furthermore, $T(A)$ separates the points of $K$. It follows from Stone-Weierstrass theorem (see $[1$, Theorem 8.3]) that $T(A)$ is (norm) dense in $C(K)$, and so $T(A)=\overline{T(A)}=C(K)$ since $T(A)$ is closed. This shows that $T$ is surjective, as required.

Theorem 3.5. If $A$ is a Banach $\ell$-space, then there exists a compact Hausdorff topological space $K$ such that $(Z(A), \circ)$ and $(C(K), \cdot)$ are isometrically and algebraically $\ell$-isomorphic as $A M$-f-algebras.

Proof. By Theorem 2.4, $Z(A)$ is an $A M$ - $f$-algebra with unit, the multiplicative identity $I$. Hence, by Theorem 3.4, there exists a compact Hausdorff topological space $K$ such that $Z(A)$ and $C(K)$ are isometrically $\ell$-isomorphic. As observed in the proof of Theorem 3.4, the $\ell$-isomorphism maps the multiplicative identity $I$ of $Z(A)$ onto the multiplicative identity 1 of $C(K)$. It follows from Corollary 5.5 of [8] that this $\ell$-isomorphism is also algebraic. Therefore, we have $(Z(A), \circ) \cong(C(K), \cdot)$, as required.

Definition 3.6. An algebra $A$ is called faithful if, for each $a \in A, A a=a A=\{0\}$ implies that $a=0$.

Before presenting the main result of this section, we recall that $Z(A) \subseteq \operatorname{Orth}(A) \subseteq$ $M(A)$ holds for every Archimedean $f$-algebra $A$ and, in the case that $A$ is a Banach $\ell$-space, $Z(A)=\operatorname{Orth}(A) \subseteq M(A)$.

Theorem 3.7. If $(A, *)$ is a faithful Banach algebra, then the following are equivalent.

(1) $(A, *)$ is an AM-f-algebra. 
(2) The representation of $(A, *)$ into $M(A)$ is a contractive algebraic $\ell$-isomorphism of $(A, *)$ into $(Z(A), \circ)$.

(3) $(A, *)$ can be identified with a closed $\ell$-subalgebra of $(C(K), \cdot)$ for some compact Hausdorff topological space $K$.

Proof. $(1) \Rightarrow(2)$ Suppose that $(A, *)$ is an $A M$-f-algebra and Define the representation $R:(A, *) \rightarrow(Z(A), \circ) \subseteq M(E)$ by $R(a)=\pi_{a}$, where $\pi_{a} b=a * b, b \in A$. For each $a \in A$, the equation $\pi_{a} b=a * b$ defines a bounded linear operator on $A$; for,

$$
\left\|\pi_{a} b\right\|=\|a * b\| \leq\|a\|\|b\| .
$$

Moreover, since $A$ is an $f$-algebra, if $a \perp b$ in $A$, then $a * c \perp b$ for all $c \in A$; that is, $\pi_{a} c \perp b$. This shows that $\pi_{a} \in Z(A)$ for all $a \in A$, as $A$ is a Banach $\ell$-space. In other words, we have $R(A) \subseteq Z(A)$.

Suppose that $a, b \in A$. Then

$$
\pi_{a \vee b} c=(a \vee b) * c=a * c \vee b * c=\pi_{a} c \vee \pi_{b} c
$$

for all $c \in A^{+}$, which shows that $R(a \vee b)=R a \vee R b$; that is, $R$ is an $\ell$-homomorphism. Moreover, $R$ is an algebra homomorphism; for, by the associativity of $A$,

$$
\pi_{a * b} c=(a * b) * c=a *(b * c)=a *\left(\pi_{b} c\right)=\pi_{a}\left(\pi_{b} c\right)=\left(\pi_{a} \circ \pi_{b}\right) c
$$

for all $c \in A$. Thus $R(a * b)=R a \circ R b$.

Since $A$ is faithful, if $R a=0$ for all $a \in A$, then $\pi_{a} b=a * b=0$ for all $b \in A$, which implies that $a=0$. Hence $R$ is injective. This proves that $R$ is an algebraic $\ell$-isomorphism.

$(2) \Rightarrow(3)$ By Proposition 3.5, $(Z(A), \circ) \cong(C(K), \cdot)$ for some compact Hausdorff topological space $K$. Hence, since $A$ is an arbitrary Banach $\ell$-algebra, $(A, *)$ is isometrically and algebraically $\ell$-isomorphic to a closed $\ell$-subalgebra of a $(C(K), \cdot)$ algebra by Theorem 3.4.

$(3) \Rightarrow(1)$ Suppose that $D$ is a closed $\ell$-subalgebra of $(C(K), \cdot)$. On the other hand, $(A, *)=(D, \cdot)$ by the hypothesis, from which the result follows.

4. The identification of $C_{b}(L, Z(A))$. We consider the center $Z\left(C_{0}(L, A)\right)$ of the space $C_{0}(L, A)$ of all continuous mappings from $L$ into $A$ vanishing at infinity, where $(L, \xi)$ is a locally compact Hausdorff topological space and $A$ is a Banach $\ell$-space. The pair $\left(C_{0}(L, A), \cdot\right)$ is a Banach $\ell$-space with respect to the natural partial ordering and the supremum norm. Moreover, it is a Banach $f$-algebra whenever $A$ is so. Suppose that $\mathcal{L}_{b}(A)$ is endowed with the strong operator topology $\tau$, and that $C_{b}\left(L, \mathcal{L}_{b}(A)\right)$ denotes the space of all norm bounded continuous mappings from $(L, \xi)$ into $\left(\mathcal{L}_{b}(A), \tau\right)$. It has been proved in [7, Theorem 6.2] that $C_{b}\left(L, \mathcal{L}_{b}(A)\right)$ can be identified with a subalgebra of $\mathcal{L}_{b}\left(C_{0}(L, A)\right)$. Moreover, this identification is an isometric algebraic $\ell$-isomorphism. The following result shows that $C_{b}(L, Z(A))$ can be identified with the center $Z\left(C_{0}(L, A)\right)$ of $C_{0}(L, A)$.

We consider the center $Z\left(C_{0}(L, A)\right)$ of the space $C_{0}(L, A)$ of all continuous mappings from $L$ into $A$ vanishing at infinity, where $(L, \xi)$ is a locally compact Hausdorff topological space and $A$ is a Banach $\ell$-space. The pair $\left(C_{0}(L, A), \cdot\right)$ is a Banach $\ell$-space with respect to the natural partial ordering and the supremum norm. Moreover, it is a Banach $f$-algebra whenever $A$ is so. Suppose that $\mathcal{L}_{b}(A)$ is endowed 
with the strong operator topology $\tau$, and that $C_{b}\left(L, \mathcal{L}_{b}(A)\right)$ denotes the space of all norm bounded continuous mappings from $(L, \xi)$ into $\left(\mathcal{L}_{b}(A), \tau\right)$. Ercan and Wickstead have proved in $\left[7\right.$, Theorem 6.2] that $C_{b}\left(L, \mathcal{L}_{b}(A)\right)$ can be identified with a subalgebra of $\mathcal{L}_{b}\left(C_{0}(L, A)\right)$. Moreover, this identification is an isometric algebraic $\ell$-isomorphism. The following result shows that $C_{b}(L, Z(A))$ can be identified with the center $Z\left(C_{0}(L, A)\right)$ of $C_{0}(L, A)$.

Theorem 4.1. Let $L$ and $A$ be as above, and suppose that $\mathcal{L}_{b}(A)$ is endowed with the strong operator topology. Then $C_{b}(L, Z(A))$ and $Z\left(C_{0}(L, A)\right)$ are isometrically and algebraically $\ell$-isomorphic as $A M$ - $f$-algebras.

Proof. We consider the mapping $T: C_{b}(L, Z(A)) \rightarrow Z\left(C_{0}(L, A)\right)$ defined by $T(\phi)=\pi_{\phi}$ for each $\phi \in C_{b}(L, Z(A))$, where $\pi_{\phi} f(x)=\phi(x)(f(x))$ for all $f \in C_{0}(L, A)$ and $x \in L$.

If $\phi \in C_{b}(L, Z(A))$, then, for each $0 \leq f \in C_{0}(L, A)$,

$$
\left\|\pi_{\phi} f\right\|=\sup _{x \in L}\|\phi(x)(f(x))\| \leq\|\phi\|\|f\|,
$$

where $\|\phi\|=\sup _{x \in L}\|\phi(x)\|$, which implies that $\left\|\pi_{\phi}\right\| \leq\|\phi\|$. Hence $T$ is bounded. Moreover, if $f \wedge g=0$ in $C_{0}(L, A)$, then it follows immediately that $\pi_{\phi} f \wedge g=0$ in $C_{0}(L, A)$. This shows that $\pi_{\phi} \in Z\left(C_{0}(L, A)\right)$ for each $\phi \in C_{b}(L, Z(A))$.

It is routine to show that $T$ is an algebraic $\ell$-isomorphism. We show only that $T$ is surjective. Suppose that $\Phi \in Z\left(C_{0}(L, A)\right)$. For $x \in L$ and $f \in C_{0}(L, A)$, the value $(\Phi f)(x)$ is unambiguously defined by the value of $f(x)$ (for, if $f_{1}(x)=f(x)$ for some $f_{1} \in C_{0}(L, A)$, then, by linearity,

$$
\left.\left|\left(\Phi\left(f_{1}-f\right)\right)(x)\right| \leq\|\Phi\| \mid\left(f_{1}-f\right)\right)(x) \mid=0,
$$

and so $\left.\Phi f_{1}(x)=\Phi f(x)\right)$.

Let $f \in C_{0}(L, A), x \in L$ and define a mapping $\varphi: L \rightarrow Z\left(C_{0}(L, A)\right)$ by $\varphi(x) f(x)=\Phi f(x)$, where $\Phi=\pi_{\varphi}$; i.e., $\varphi(x) f(x)=\pi_{\varphi} f(x)$. In the same way above it is easily seen that $\varphi(x) \in Z\left(C_{0}(L, A)\right)$ and that $\varphi$ is unambiguously defined. It remains to show that $\varphi \in C_{b}(L, Z(A))$. As $\Phi \in Z\left(C_{0}(L, A)\right)$, we have $\Phi\left(C_{0}(L, A)\right) \subseteq C_{0}(L, A)$; in other words, $\pi_{\varphi}\left(C_{0}(L, A)\right) \subseteq C_{0}(L, A)$. It follows from Theorem 6.1 of [7] that $\varphi$ is norm bounded and continuous with respect to the strong operator topology. So $\varphi \in C_{b}(L, Z(A))$, as required.

The isometric property follows from $\left\|\pi_{\phi}\right\|=\|\phi\|$, as follows. As already established, $\left\|\pi_{\phi}\right\| \leq\|\phi\|$. Since

$$
\|\phi(x)\|=\sup _{a \in A}\|\phi(x)(a)\|
$$

we have that, for all $x \in L$,

$$
\|\phi(x)\|_{f(L)}=\sup _{f(x) \in A}\|\phi(x)(f(x))\|=\sup _{f(x) \in A}\left\|\pi_{\phi} f(x)\right\| \leq\left\|\pi_{\phi}\right\|
$$

and so $\|\phi\|_{f(L)} \leq\left\|\pi_{\phi}\right\|$. It follows that $\left\|\pi_{\phi}\right\|=\|\phi\|$; that is, $\|T(\phi)\|=\|\phi\|$.

Since $C_{0}(L, A)$ is a Banach $\ell$-space, $Z\left(C_{0}(L, A)\right)$ is an $A M$-f-algebra with unit by Theorem 2.4. We therefore have $Z\left(C_{0}(L, A)\right) \cong C_{b}(L, Z(A))$ as $A M$-f-algebras, as required. 
Remark 4.2. (1) The mapping $1: L \rightarrow Z(A)$, defined by $1(x)=I$ for all $x \in L$ is a multiplicative identity of $C_{b}(L, Z(A))$, and so is a norm order unit in $C_{b}(L, Z(A))$ as observed in Remark 2.5, where $I$ is the identity operator in $Z(A)$.

(2) If we denote the identity operator in $Z\left(C_{0}(L, A)\right)$ by $I I$ (that is, $I I(f)=f$ for all $\left.f \in C_{0}(L, A)\right)$, then $\pi_{1}=I I$ since

$$
\pi_{1} f(x)=1(x)(f(x))=I(f(x))=f(x)
$$

for all $x \in L$, and so $\pi_{1} f=f$ for all $f \in C_{0}(L, A)$. It follows that $T(1)=I I$; in other words, $T$ maps the multiplicative identity of $C_{b}(L, Z(A))$ into that of $Z\left(C_{0}(L, A)\right)$.

Corollary 4.3. For every locally compact Hausdorff topological space $L, Z\left(C_{0}(L)\right) \cong$ $C_{b}(L)$ as AM-f-algebras.

\section{REFERENCES}

[1] Aliprantis, C. D. and Burkinshaw, O. (1981). Principles of Real Analysis. Elsevier NorthHolland, New York and Oxford.

[2] Aliprantis, C. D. and Burkinshaw, O. (1985). Positive Oparators. Academic Press.

[3] Bernau, S. J. and Huijsmans, C. B. (1990). Almost $f$-algebras and $d$-algebras. Math. Proc. Cambridge Philos. Soc. 107, 287-308.

[4] Birkhoff, G. (1967). Lattice Theory. Amer. Math. Soc. Colloq. Publ. No. 25.

[5] Birkhoff, G. and Pierce, R. S. (1956). Lattice-ordered rings. An. Acad. Brasil. Ciénc. 28, 41-49.

[6] Conwayi, J. B. (1985). A Course in Funtional Analysis. Spinger-Verlag New York nc.

[7] Ercan, Z. and Wickstead, A. W. (1996). Banach lattices of continuous Banach lattices-valued functions. J. Math. Anal. Appl. 198, 121-136.

[8] Huijsmans, C. B. and de Pagter, B. (1984). The order bidual of lattice algebras. J. Funct. Anal. $59,41-64$.

[9] Kakutani, S. (1941) Concrete represantation of abstract $M$-spaces. Ann. of Mat. 42, 994-1024.

[10] Krein, M. and Krein, S. (1940). On an inner characteristic of the set of all continuos functions definrd on a bicompact Hausdorff spaces. C. R. (Dokl.) Acad. Sci. URSS (N.S.) 27, 427-430.

[11] Krein, M. and Krein, S. (1943). Sur l'espace des fonctions continues définies sur un bicompact de on a Hausdorff et ses sous-espaces semi-ordonnés. Mat. Sb. 13, 18-38.

[12] Krein, M. and Milman, D. (1940). On extreme points of regular convex sets. Studia Math. 9, 133-138.

[13] Kudláček, V. (1962). On some types of $\ell$-rings. Sborni Vysokého Učeni Techn v Brně 1-2, 179-181.

[14] Luxemburg, W. A. J. and Zaanen, A. C. (1971). Riesz Spaces I. North-Holland.

[15] Martignon, L. (1980). Banach $f$-algebras and Banach lattice algebras with unit. Bol. Soc. Bras. Mat. 11, 11-18.

[16] Schefold, H. H. (1974). Banach Lattices and Positive Operators. Spinger-Verlag Berlin Heidelberg New York.

[17] Wickstead, A. W. (1977). Representation and duality of multiplication operators on Archimedean Riesz spaces. Compositio Math. 35, 225-238.

[18] Yilmaz, R. and Rowlands, K. (2006). On orthomorphisms, quasi-orthomorphisms and quasimultipliers. J. Math. Anal. Appl. 313, 120-131.

[19] Zaanen, A. C. (1983). Riesz Spaces II. North-Holland.

[20] Zaanen, A. C. (1997). Introduction to Operator Theory in Riesz Spaces. Springer. 